\title{
Segmentation of Intra-retinal Layers in 3D Optic Nerve Head Images
}

\author{
Chuang Wang ${ }^{1(凶)}$, Yaxing Wang ${ }^{2}$, Djibril Kaba ${ }^{1}$, Haogang $\mathrm{Zhu}^{3}$, You $\mathrm{Lv}^{4}$, \\ Zidong Wang ${ }^{1}$, Xiaohui $\mathrm{Liu}^{1}$, and Yongmin $\mathrm{Li}^{1}$ \\ 1 Department of Computer Science, Brunel University London, Uxbridge, UK \\ Chuang. Wang@brunel.ac.uk \\ 2 Tongren Hospital, Beijing, China \\ 3 Moorfields Eye Hospital NHS Foundation Trust, London, UK \\ 4 School of Computer and Engineering, \\ Nanjing University of Science and Technology, Nanjing, China
}

\begin{abstract}
Spectral-Domain Optical Coherence Tomography (SD-OCT) is a non-invasive imaging modality, which provides retinal structures with unprecedented detail in 3D. In this paper, we propose an automated segmentation method to detect intra-retinal layers in SD-OCT images around optic nerve head acquired from a high resolution RTVue-100 SDOCT (Optovue, Fremont, CA, USA). This method starts by removing all the OCT imaging artifacts including the speckle noise and enhancing the contrast between layers using the 3D nonlinear anisotropic. Afterwards, we combine the level set method, k-means and MRF method to segment three intra-retinal layers around optical nerve head. The segmentation results show that our method can effectively delineate the surfaces of the retinal tissues in the noisy $3 \mathrm{D}$ optic nerve head images. The signed and unsigned significant differences between the segmentation results and the ground truth over optic nerve head B-scans are $1.01 \pm 1.13$ and $1.93 \pm 2.21$
\end{abstract}

\section{Introduction}

Optical Coherence Tomography (OCT) is a powerful biomedical tissue-imaging modality, which can provide wealthy information, such as structure information, blood flow, elastic parameters, change of polarization state and molecular content [9]. Therefore, this imaging tool has been increasingly useful in diagnosing eye diseases, such as glaucoma, diabetic retinopathy and age-related macular degeneration. These diseases are known to be the most common causes of blindness in the developed countries according to the World Heath Organization (WHO) survey [15]. In order to help ophthalmologists to perform more accurately and efficiently the diagnosis of eye diseases, several medical image processing techniques are applied to extract some useful information from OCT data, such as retinal layers, retinal vessels, retinal lesions, optic nerve head, optic cup and neuroretinal rim. In this work, we focus on the intra-retinal layer segmentation of $3 \mathrm{D}$ retinal images obtained from around the macular and the optic disc head. 
There are two main reasons for intra-retinal layer segmentation [7]. First, the morphology and thickness of each intra-retinal layer are important indicators for assessing the presence of ocular disease. For example, the thickness of the nerve fiber layer is an important indicator of glaucoma. Second, intra-retinal layer segmentation improves the understanding of the pathophysiology of the systemic diseases. For instance, the damage of the nerve fiber layer can provide the indication of brain damages [7].

However, it is time consuming or even impossible for ophthalmologist to manually label each layers, specifically for the macular images with the complicated 3D layer structures. Therefore, a reliable automated method for layer segmentation is attractive in computer aided-diagnosis. 3D OCT layer segmentation is a challenging problem, and there has been significant effort in this area over the last decade. A number of different approaches are developed to do the segmentation, however, no typical segmentation method can work equally well on different 3D retinal images collected from different imaging modalities.

For most of the existing 3D segmentation approaches, a typical two-step process is adopted. The first step is de-noising to remove the speckle noises and enhance the contrast between layers (usually with 3D anisotropic diffusion method, 3D median filter, 3D Gaussian filter or 3D wavelet transform). The second step is to segment the layers according to the characteristics of the images, such as shapes, textures or intensities.

Snake based methods [11] attempt to minimize the energy of a sum of internal and external energy of the current contour. These methods work well on images with high contrast, high gradient and smooth boundary between the layers. However, the performance is adversely affected by the blood vessel shadows, other morphological features of the retinal, or irregular layer shapes. Zhu et al. [20] proposed a Floatingcanvas method to segment 3D intra-retinal layers from 3D optic nerve head images. This method can produce relatively smooth layer surface, however, it is sensitive to the low gradient between layers. Yazdanpanah et al. [18] proposed an active contour method, incorporating with circular shape prior information, to segment intra-retinal layer from 3D OCT image. This method can effectively overcome the affects of the blood vessel shadows and other morphological features of the retinal, however it cannot work well on images with irregular layer shapes.

Pattern recognition based techniques perform the layer segmentation by using boundary classifier, which is used to assign each voxel to layer boundary and non boundary. The classifier is obtained through a learning process supervised by reference layer boundaries. Fuller et al. [5] designed a multi-resolution hierarchical support vector machines (SVMs) to segment OCT retinal layer. Compared to other methods, the segmentation accuracy is slightly lower with 6 pixels of line difference and $8 \%$ of the thickness difference. A column classification algorithm was proposed by Michael et al. [1] to segment the intra-retinal layers from 3D optic nerve head images. Lang et al. [12] trained a random forest classifier to segment retinal layers from macular images. However, the performance of the pattern recognition based techniques are highly relayed on training sets. 
Graph based methods are aimed to find the global minimum cut of the segmentation graph, which is constructed with regional term and boundary term. Garvin [6] proposed a 3D graph search method by constructing geometric graph with edge and regional information and five intra-retinal layers were successfully segmented. This method was extended in [4], which combined graph theory and dynamic programming to segment the intra-retinal layers and eight retinal layer boundaries were located. Although these methods provide good segmentation accuracy, they can not segment all layer boundaries simultaneously and have slow processing speed. Lee et al. [13] proposed a parallel graph search method to overcome these limitations. Besides, a fast multi scale 3-D graph algorithm was developed to segment the intra-retinal surfaces for 3D optic nerve head images by Lee et al. [14]. Kafieh et al. [10] proposed the coarse grained diffusion maps relying on regional image texture without requiring edge based image information and ten layers were segmented accurately. However, this method has high computational complexity and does not work well for abnormal images.

In this paper, we propose an automatic approaches to segmenting intraretinal layers from optic nerve head images. Markov Random Field (MRF) and level set method are used to segment retinal layers for 3D optic nerve head images. Firstly, the nonlinear anisotropic diffusion approach is applied to denoise the optic nerve head images and enhance the contrast between intra-retinal layers. Then, level set method is used to segment the retinal layer area. After that, the initial segmentation is obtained by using the k-means method. Because of the inhomogeneity and blood vessel shadows, the k-means method cannot segment all layers well. Therefore, MRF method is used to improve the initial segmentation through iteration until it converges or reaches the maximum iteration.

This paper is organised as follows. A detailed description of the proposed method for 3D OCT optic nerve head images is presented in Sect. 2. The experimental results are shown in Sect. 3. Finally, conclusions are drawn in Sect. 4.

\section{Optic Nerve Head Intra-retinal Layer Segmentation}

Figure 1 shows the process of layer segmentation for 3D optic nerve head images. The intra-retinal layers for optic nerve head images are segmented by two major steps: preprocessing step and layer segmentation step. During the preprocessing step, the nonlinear anisotropic diffusion approach is applied to 3D optic nerve head images to remove speckle noise and enhance the contrast between retinal layers and background. Intra-retinal layers are segmented by two major steps: preprocessing step and layer segmentation step. At the second step, four intraretinal layers are segmented by using the combination methods, which include level set method, K-means cluster and MRF.

\subsection{Preprocessing}

During the OCT imaging of the retinal, the speckle noise is generated simultaneously. The conventional anisotropic diffusion approach (Perona-Malik) [8] 


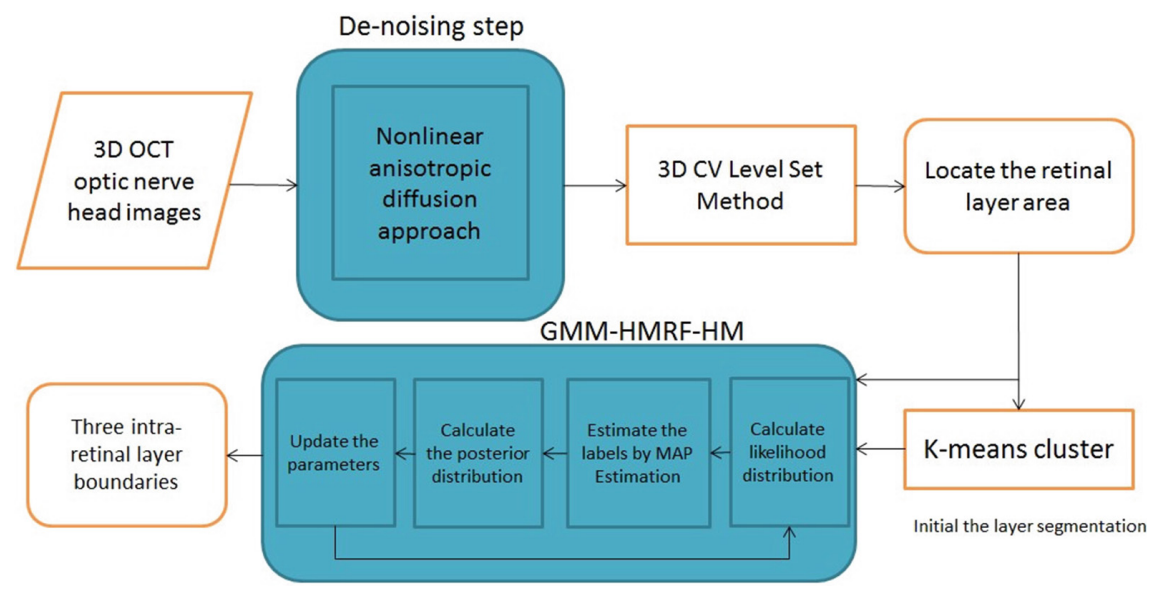

Fig. 1. Block diagram of retinal layers segmentation process for 3D optic nerve head images.

is used to remove the speckle noise and sharpen the boundaries of the retinal layers. The nonlinear anisotropic diffusion filter is defined as:

$$
\frac{\partial}{\partial I(\bar{x}, t)}=\operatorname{div}[c(\bar{x}, t) \nabla I(\bar{x}, t)]
$$

where the vector $\bar{x}$ represents $(\mathrm{x}, \mathrm{y}, \mathrm{z})$ and $\mathrm{t}$ is the process ordering parameter. $I(\bar{x}, t)$ is macular voxel intensity. $c(\bar{x}, t)$ is the diffusion strength control function, which is depended on the magnitude of the gradient of the voxel intensity. The function of $c(\bar{x}, t)$ is:

$$
c(\bar{x}, t)=\exp \left(-\frac{|\nabla I(\bar{x}, t)|^{2}}{\kappa}\right)
$$

where $\kappa$ is a constant variable chosen according to the noise level and edge strength. Finally, the voxel intensities are updated by the following formulate:

$$
I(t+\triangle t)=I(t)+\triangle t \frac{\partial}{\partial t} I(t)
$$

\subsection{Vitreous and Choroid Boundaries Segmentation}

The level set method has been extensively applied to image segmentation area. There are two major classes of the level set method: region-based models and edge-based models. The edge-based models use local edge information to direct active contour to the object boundaries, while the region-based models use a certain descriptor to identify each region of interest to guide the active contour to the desired boundary. In this study, the classical region based Chan-Vese model [3] is used to locate the boundaries of vitreous and choroid layer from 
$3 \mathrm{D}$ optic nerve head images because it works well when there is large gradient between retinal tissues and background.

The energy function of the Chan-Vese method is defined as:

$$
\begin{aligned}
E(\phi)= & \lambda_{1} \int_{\text {outside }(C)}\left(I(X)-c_{1}\right)^{2} d X+ \\
& \lambda_{2} \int_{\text {inside }(C)}\left(I(X)-c_{2}\right)^{2} d X+\nu \int_{\Omega}|\nabla H(\phi(X))| d X
\end{aligned}
$$

where $\lambda_{1}, \lambda_{2}$ are constant parameters determined by the user, $\nu$ is set to zero. In addition, outside $(C)$ and inside $(C)$ indicate the region outside and inside the contour $C$, respectively, and $c_{1}$ and $c_{2}$ are the average image intensity of outside $(C)$ and inside $(C) . \phi$ is defined as a signed distance function (SDF) that is valued as positive inside $C$, negative outside $C$, and equal to zero on $C$. The regularization term Heaviside function $H$ and the average intensities $c_{1}$ and $c_{2}$ are formulated as:

$$
\begin{gathered}
H(\phi(X))=\frac{1}{2}\left(1+\frac{2}{\pi} \arctan \left(\frac{X}{\epsilon}\right)\right) \\
c_{1}=\frac{\int_{\Omega} I(X) H(\phi(X)) d X}{\int_{\Omega} H(\phi(X)) d X} \quad c_{2}=\frac{\int_{\Omega} I(X)(1-H(\phi(X))) d X}{\int_{\Omega}(1-H(\phi(X))) d X}
\end{gathered}
$$

In calculus of variations [2], minimizing the energy functional of $E(\phi)$ with respect to $\phi$ by using gradient decent method:

$$
\frac{\partial \phi}{\partial t}=-\frac{\partial E(\phi)}{\partial \phi}
$$

where $\frac{\partial E(\phi)}{\partial \phi}$ is the Gâteaux derivative [2] of the energy function $E(\phi)$. The equation of (4) is derived by using Euler-Lagrange equation [16], which gives us the gradient flow as follow:

$$
\frac{\partial \phi}{\partial t}=-\left\{\lambda_{1}\left(I(X)-c_{1}\right)^{2}-\lambda_{2}\left(I(X)-c_{2}\right)^{2}\right\} H(\phi(X))
$$

\subsection{RNFL and RPE Layers Segmentation}

After locating the boundaries of the vitreous and choroid layers, we define a region that includes all the layers. In order to reduce the computation load and increase the speed of the segmentation, we cut the retinal area out alone the top and bottom layer boundaries. The K-means cluster is used to initialize the shrinked data $I_{s}$ into $k$ classes $S=\left\{S_{1}, S_{2}, \ldots, S_{k}\right\}$ :

$$
X=\arg \min _{S} \sum_{i=1}^{k} \sum_{I_{s}(p) \in S_{i}}\left\|I_{s}(p)-\mu_{i}\right\|^{2}
$$

where $\mu_{i}$ is the mean intensity in $S_{i}$.

However, the k-means cluster fails to accurately locate all the layers due to the blood vessel shadows and intensity inhomogeneities. Therefore, MRF is applied to update the initial input $X$ through iteration until it converges or reaches the maximum iteration. There are four main steps of this method: first we 
calculate the likelihood distribution according the initialization information; then we estimate the labels using MAP method; after that, the posterior distribution is calculated and the parameter set is updated.

The MRF has been first introduced to segment Brain MR images [19]. Given a 3D image $Y=\left(y_{1}, \ldots, y_{i}, \ldots, y_{N}\right)$, where $N$ is the total number of voxels and each $y_{i}$ is a grey level voxel intensity, and $X=\left(x_{1}, \ldots, x_{i}, \ldots x_{N}\right)\left(x_{i} \in L\right)$ is corresponding initial label of each voxel of the image. For example $L=\{0,1\}$, the image is segmented into two regions. The RNFL and RPE layers are segmented by using MRF method. Here, we set $L=\{0,1,2,3\}$.

EM algorithm is used to estimate the parameter set $\Theta=\left\{\theta_{l} \mid l \in L\right\}$. It is assumed that the voxel intensity $y_{i}$ follows the gaussian mixture model with $g$ components parameters $\theta_{i}$ given the label $x_{i}$ :

$$
P\left(y_{i} \mid x_{i}\right)=G_{m i x}\left(y_{i} ; \theta_{i}\right)
$$

Based on the conditional independence assumption of $y$, the joint liked probability can be expressed as:

$$
P(Y \mid X)=\prod_{i=0}^{N} P\left(y_{i} \mid x_{i}\right)=\prod_{i=0}^{N} G_{m i x}\left(y_{i} ; \theta_{i}\right)
$$

Start: The initial GMM with g components parameter set $\Theta^{0}$ is learned from the labels $X$ and image data $Y$. The parameters can be expressed as:

$$
\theta_{l}=\left(\mu_{l, 1}, \sigma_{l, 1}, \omega_{l, 1}\right), \ldots,\left(\mu_{l, g}, \sigma_{l, g}, \omega_{l, g}\right)
$$

And the weighted probability of the GMM is:

$$
\begin{aligned}
G_{m i x}\left(y ; \theta_{l}\right) & =\sum_{c=1}^{g} \omega_{l, c} G\left(y ; \mu_{l, c}, \sigma_{l, c}\right) \\
& =\sum_{c=1}^{g} \frac{1}{\sqrt{2 \pi \sigma_{l, c}^{2}}} \exp \left(-\frac{\left(y-\mu_{l, c}\right)^{2}}{2 \sigma_{l, c}^{2}}\right)
\end{aligned}
$$

E-step: At the $t^{t h}$ iteration, we can obtain the parameters $\Theta^{t}$, and the conditional expectation can be deduced as:

$$
\begin{aligned}
Q\left(\Theta \mid \Theta^{t}\right) & =E\left[\ln P(X, Y \mid \Theta) \mid Y, \Theta^{t}\right] \\
& =\sum_{X \in L} P\left(X \mid Y, \Theta^{t}\right) \ln P(X, Y \mid \Theta)
\end{aligned}
$$

where $L$ is the set of all possible labels, and $P(X, Y \mid \Theta)$ can be rewritten as:

$$
P(X, Y \mid \Theta)=P(X \mid Y) P(Y \mid \Theta)
$$

M-step: Next parameter set $\Theta^{t+1}$ is estimated through maximizing $Q\left(\Theta \mid \Theta^{t}\right)$ :

$$
\Theta^{t+1}=\arg \max _{\Theta} Q\left(\Theta \mid \Theta^{t}\right)
$$

The next let $\Theta^{t+1} \rightarrow \Theta^{t}$, and repeat from E-step. 
It is assumed that the prior probability can be written as:

$$
P(X)=\frac{1}{Z} \exp (-U(X))
$$

where $U(X)$ is the prior energy function. We also assume that:

$$
\begin{aligned}
P(Y \mid X, \Theta) & =\prod_{i} P\left(y_{i} \mid x_{i}, \theta_{x_{i}}\right)=\prod_{i} G_{m i x}\left(y_{i} ; \theta_{x_{i}}\right) \\
& =\frac{1}{Z^{\prime}} \exp (-U(Y \mid X))
\end{aligned}
$$

Under these assumptions, the MRF Algorithm [17] is given below:

1. Initialise the parameter set $\Theta^{0}$.

2. Calculate the likelihood distribution $P^{t}\left(y_{i} \mid x_{i}, \theta_{x_{i}}\right)$.

3. Estimate the labels by MAP estimation using the current parameter $\Theta^{t}$ :

$$
\begin{aligned}
X^{(t)} & =\underset{X \in L}{\arg \max }\left\{P\left(Y \mid X, \Theta^{(t)}\right) P(X)\right\} \\
& =\underset{X \in L}{\arg \min }\left\{U\left(Y \mid X, \Theta^{(t)}\right)+U(X)\right\}
\end{aligned}
$$

Given $\mathrm{X}$ and $\Theta$, the likelihood energy (also called unitary potential) is

$$
U(Y \mid X, \Theta)=\sum_{i=1}^{N} U\left(y_{i} \mid x_{i}, \Theta\right)=\sum_{i=1}^{N}\left[\frac{\left(y_{i}-\mu_{x_{i}}\right)^{2}}{2 \sigma_{x_{i}}^{2}}+\ln \sigma_{x_{i}}\right]
$$

The prior energy function $U(X)$ is defined as:

$$
U(X)=\sum_{c \in C} V_{c}(X)
$$

where $V_{c}(X)$ is the clique potential and $\mathrm{C}$ is the set of all possible cliques. For 3D image, we assume that one voxel has at most 32-neighborhood. The clique potential is defined as:

$$
V_{c}\left(x_{i}, x_{j}\right)=\beta\left(1-I_{x_{i}, x_{j}}\right)
$$

where $\beta$ is a constant variable coefficient set to $1 / 6$. The function $I_{x_{i}, x_{j}}$ is:

$$
I_{x_{i}, x_{j}}=\left\{\begin{array}{l}
1, \text { if } x_{i}=x_{j} \\
0, \text { if } x_{i} \neq x_{j}
\end{array}\right.
$$

Firstly, the initial estimation $X^{0}$ is calculated from the previous loop of the EM algorithm. Then, an iterative algorithm is developed to estimate the $X^{k+1}$ provided $X^{k}$ until $U(Y \mid X, \Theta)+U(X)$ converges or reaches the maximum $k$.

4. Calculate the posterior distribution for all $l \in L$ and voxels $y_{i}$ using Bayesian rule:

$$
P^{t}\left(l \mid y_{i}\right)=\frac{G_{m i x}\left(y_{i} ; \theta_{l}\right) P\left(l \mid x_{N_{i}}^{t}\right)}{P^{t}\left(y_{i}\right)}
$$


Table 1. Signed and unsigned mean and SD difference between the ground truth and the proposed segmentation results for the four surfaces, respectively.

\begin{tabular}{l|l|l}
\hline Surface & Signed difference $($ mean $1 \pm \mathrm{SD})$ & Unsigned difference $($ mean $\pm \mathrm{SD})$ \\
\hline 1 & $-0.42 \pm 0.65$ & $0.83 \pm 0.79$ \\
\hline 2 & $1.01 \pm 1.13$ & $1.43 \pm 1.98$ \\
\hline 3 & $0.51 \pm 1.14$ & $1.02 \pm 1.62$ \\
\hline 4 & $-0.9 \pm 1.53$ & $1.93 \pm 2.21$ \\
\hline
\end{tabular}

where the conditional probability $P\left(l \mid x_{N_{i}}^{t}\right)$ :

$$
P\left(l \mid x_{N_{i}}^{t}\right)=\frac{1}{Z} \exp \left(-\sum_{j \in N_{i}} V_{c}\left(l, x_{j}^{t}\right)\right)
$$

$x_{N_{i}}^{t}$ is the neighborhood configuration of $x_{i}^{t}$, and the intensity distribution function is:

$$
P^{t}\left(y_{i}\right)=P\left(y_{i} \mid \theta^{t}\right)=\sum_{l \in L} G_{m i x}\left(y_{i}, \theta_{l}\right) P\left(l \mid x_{N_{i}}^{t}\right)
$$

5. Update the parameters by using $P\left(l \mid x_{N_{i}}^{t}\right)$

$$
\begin{aligned}
& \mu_{l}^{(t+1)}=\frac{\sum_{i} P^{t}\left(y_{i}\right) y_{i}}{\sum_{i} P^{t}\left(y_{i}\right)} \\
& \left(\sigma_{l}^{(t+1)}\right)^{2}=\frac{\sum_{i} P^{t}\left(y_{i}\right)\left(y_{i}-\mu_{l}^{(t+1)}\right)^{2}}{\sum_{i} P^{t}\left(y_{i}\right)}
\end{aligned}
$$

\section{Experiments}

We tested the proposed method on SD-OCT optic nerve head images obtained with RTVue-100 SD-OCT (Optovue, Fremont, CA, USA) in Moorfileds Eye Hospital. The age of the enrolled subjects ranged from 20 to 85 years. This imaging modalities protocols have been widely used to diagnose the glaucoma diseases, which provide 3D image with 16 bits per pixel and $101 \mathrm{~B}$-scans, 513 A-scans, 768 pixels in depth. Our methods successfully segmented the 4 intraretinal surfaces of all the $3 \mathrm{D}$ optical nerve head images without any segmentation failures. The signed and unsigned mean and standard deviation (SD) difference between the ground truth and the proposed segmentation results of the four surfaces are given in Table 1. In terms of the signed and unsigned differences, the first surface gives the best performance $(-0.42 \pm 0.65)$ and $(0.83 \pm 0.79)$, respectively.

Figure 2 shows two examples of three intra-retinal layers segmented results from a 3D OCT optic nerve head image which the layer 1 is retinal nerve fiber layer, layer 2 includes Ganglion Cell Layer, Inner Plexiform Layer, Inner Nuclear Layer and Outer Nuclear Layer (GCL, IPL, INL and ONL), layer 3 is retinal pigment epithelium layer. Figure 2(a) shows the 60th B-scan, which includes the optic disc region. Three examples of 3D OCT optic nerve head image layer 

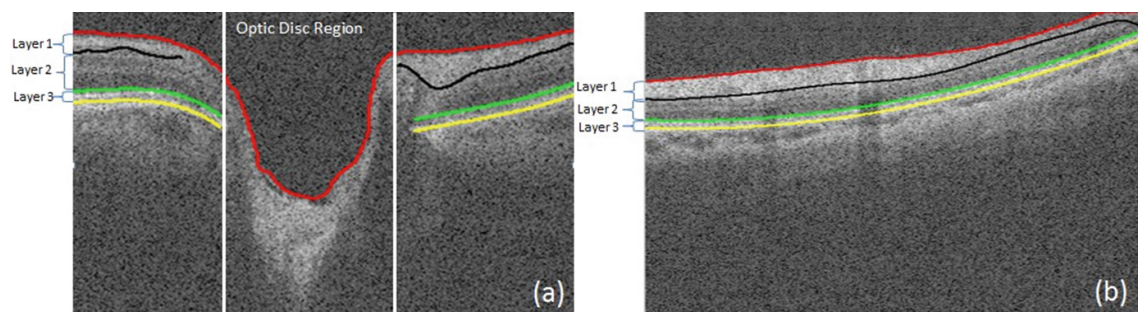

Fig. 2. Illustration of three intra-retinal layers segmented results of two cross-sectional B-scans from a 3D OCT optic nerve head image. (a) the 60th B-scan, which includes the optic disc region, (b) the 10th B-scan. Layer 1: retinal nerve fiber layer (RNFL), Layer 2 includes Ganglion Cell Layer, Inner Plexiform Layer, Inner Nuclear Layer and Outer Nuclear Layer (GCL, IPL, INL and ONL), Layer 3: retinal pigment epithelium layer (RPE).

segmented results are demonstrated in Fig. 3. In Fig. 3, four segmented layer surfaces are illustrated in 3D, and the shape of the surfaces are hypothesised to be related with eye diseases. Figure 4 illustrates the segmented results of 10 example B-scans from a segmented 3D optic nerve head image. Looking at these segmented segmentation results, our method can efficiently and accurately detect each layer of the retina in the $3 \mathrm{D}$ retinal images around the optic nerve head.
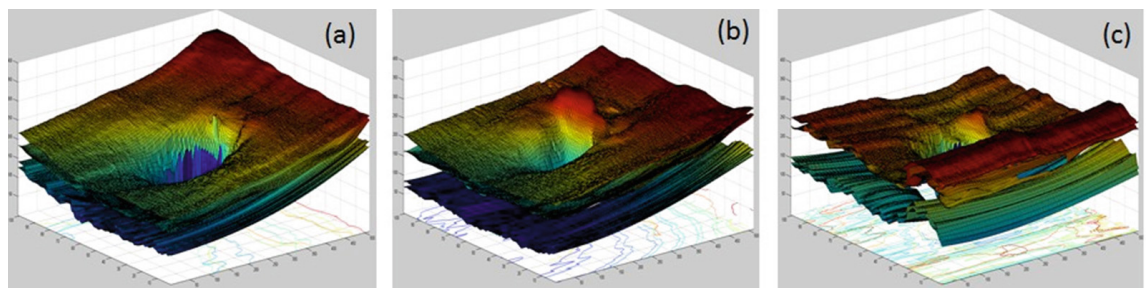

Fig. 3. Three examples of 3D OCT optic nerve head image layers segmentation results. Four segmented layer surfaces of 3 different 3D images are visualised in $3 \mathrm{D}$. The shape of the surfaces are hypothesised to be related with eye diseases.

The RNFL thickness map is useful in discriminating for glaucomatous eyes from normal eyes. Therefore, the RNFL layer thickness map is generated after the segmentation of different retinal layers. With the thickness map of RNFL, we can distinguish the glaucomatous patient from the normal subjects. Figure 5 shows two examples of the thickness map of RNFL of a healthy subject and a glaucomatous patient. In Fig. 5(a), we can observe a thick retinal nerve fiber layer, while Fig. 5(b) displays a thin retinal nerve fiber layer.

The proposed approaches are implemented on MATLAB R2011b, and the average computation time of our algorithm is $208.45 \mathrm{~s}$ for a $3 \mathrm{D}$ optic never head image on Intel (R) Core(TM) i5-2500 CPU, clock of 3.3 GHz, and 8G RAM memory. 


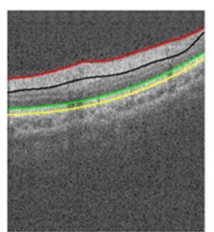

(a)

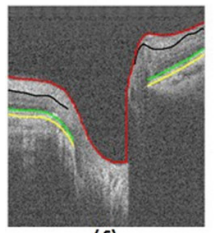

(f)

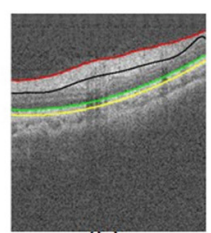

(b)

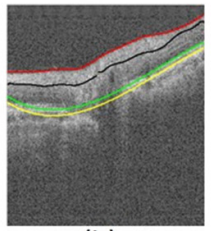

(h)

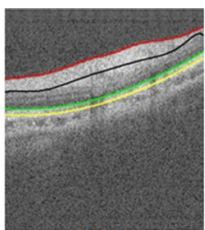

(c)

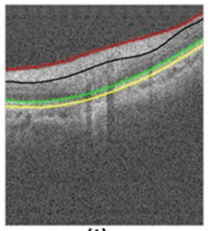

(i)

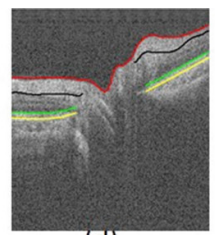

(d)

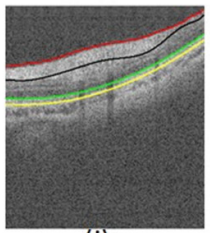

(j)

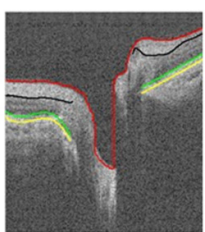

(e)

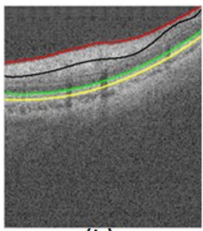

(k)

Fig. 4. Ten B-scan segmentation results from an example 3D segmented optic nerve head image, (a)-(k) are 10th, 20th, 30th, 40th, 50th, 60th, 70th, 80th, 90th, 100th B-scans, respectively. According to the segmentation results on B-scans from the 3D retinal images around the optic nerve head, the efficiency and accuracy of our method are shown.

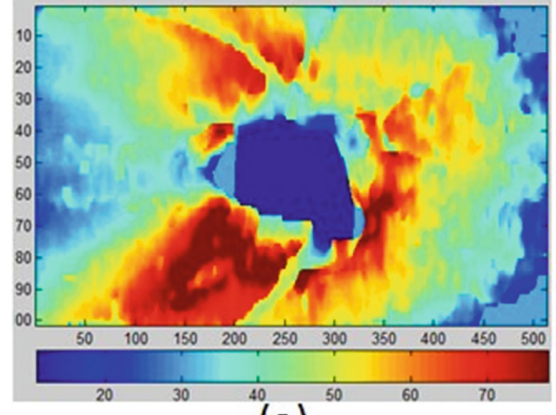

(a)

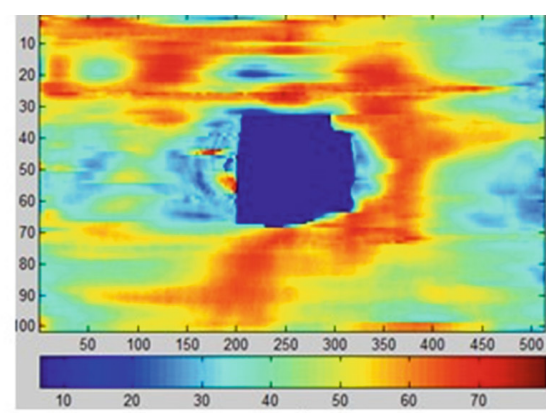

(b)

Fig. 5. The thickness maps of retinal nerve fiber layer (RNFL) from two 3D optic nerve head image examples. The RNFL thickness map is useful in discriminating for glaucomatous eyes from normal eyes. (a) a healthy subject (b) a glaucomatous patient.

\section{Conclusions and Discussions}

In this paper, an automated hybrid retinal layer segmentation method is presented for 3D optic nerve head images. This method was implemented with a typical two-staged process: de-noising step and segmentation step. The nonlinear anisotropic diffusion approach is used to filter the speckle noise and enhance the contrast between the layers as a preprocessing step.

A novel hybrid intra-retinal layer segmentation method for $3 \mathrm{D}$ optic nerve head images has been presented. This method combines the CV model based level set, k-means cluster and the Gaussian mixture model based Markov Random 
Field. The segmentation results show that our approach can detect four surfaces accurately for 3D optic nerve head images.

It seems that the segmentation process is too complicated to involve three different methods, namely the level set method, k-means cluster and MRF. However, it is difficult or even impossible to segment all the layers simultaneously by using a single method because it requires larger computation memory and longer computation time for a high volume of 3D images. Although methods such as sub-sampling are applied to reduce the volume size, some important information may lose. Conversely, a better segmentation with less computation is obtained by using our method. More specifically, the CV model based level set method first segments the volume of retinal area, the k-means cluster method initialises the volume data into $k$ regions, and the MRF method updates the initialization to overcome the artifacts such the blood vessel shadow and variation of the image intensity.

Acknowledgments. The authors would like to thank Quan Wang for providing the source of the MRF method.

\section{References}

1. Abràmoff, M.D., Lee, K., Niemeijer, M., Alward, W.L., Greenlee, E.C., Garvin, M.K., Sonka, M., Kwon, Y.H.: Automated segmentation of the cup and rim from spectral domain oct of the optic nerve head. Invest. Ophthalmol. Vis. Sci. 50(12), 5778-5784 (2009)

2. Aubert, G., Kornprobst, P.: Mathematical Problems in Image Processing: Partial Differential Equations and the Calculus of Variations, vol. 147. Springer, Heidelberg (2006)

3. Chan, T.F., Vese, L.A.: Active contours without edges. IEEE Trans. Image Process. 10(2), 266-277 (2001)

4. Chiu, S.J., Li, X.T., Nicholas, P., Toth, C.A., Izatt, J.A., Farsiu, S.: Automatic segmentation of seven retinal layers in sdoct images congruent with expert manual segmentation. Opt. Express 18(18), 19413-19428 (2010)

5. Fuller, A.R., Zawadzki, R.J., Choi, S., Wiley, D.F., Werner, J.S., Hamann, B.: Segmentation of three-dimensional retinal image data. IEEE Trans. Vis. Comput. Graph. 13(6), 1719-1726 (2007)

6. Garvin, M.K., Abràmoff, M.D., Kardon, R., Russell, S.R., Wu, X., Sonka, M.: Intraretinal layer segmentation of macular optical coherence tomography images using optimal 3-D graph search. IEEE Trans. Med. Imag. 27(10), 1495-1505 (2008)

7. Garvin, M.K., Abràmoff, M.D., Wu, X., Russell, S.R., Burns, T.L., Sonka, M.: Automated 3-D intraretinal layer segmentation of macular spectral-domain optical coherence tomography images. IEEE Trans. Med. Imag. 28(9), 1436-1447 (2009)

8. Gerig, G., Kubler, O., Kikinis, R., Jolesz, F.A.: Nonlinear anisotropic filtering of MRI data. IEEE Trans. Med. Imag. 11(2), 221-232 (1992)

9. Huang, D., Swanson, E.A., Lin, C.P., Schuman, J.S., Stinson, W.G., Chang, W., Hee, M.R., Flotte, T., Gregory, K., Puliafito, C.A., et al.: Optical coherence tomography. Science 254(5035), 1178-1181 (1991) 
10. Kafieh, R., Rabbani, H., Abramoff, M.D., Sonka, M.: Intra-retinal layer segmentation of 3D optical coherence tomography using coarse grained diffusion map. Med. Image Anal. 17(8), 907-928 (2013)

11. Kass, M., Witkin, A., Terzopoulos, D.: Snakes: active contour models. Int. J. Comput. Vis. 1(4), 321-331 (1988)

12. Lang, A., Carass, A., Hauser, M., Sotirchos, E.S., Calabresi, P.A., Ying, H.S., Prince, J.L.: Retinal layer segmentation of macular oct images using boundary classification. Biomed. Opt. Express 4(7), 1133-1152 (2013)

13. Lee, K., Abràmoff, M.D., Garvin, M.K., Sonka, M.: Parallel graph search: application to intraretinal layer segmentation of 3-D macular oct scans. In: SPIE Medical Imaging, p. 83141H (2012)

14. Lee, K., Niemeijer, M., Garvin, M.K., Kwon, Y.H., Sonka, M., Abràmoff, M.D.: Segmentation of the optic disc in 3 -D oct scans of the optic nerve head. IEEE Trans. Med. Imag. 29(1), 159-168 (2010)

15. Organization, W.H.: Coding Instructions for the WHO/PBL Eye Examination Record (version iii). WHO, Geneva (1988)

16. Smith, B., Saad, A., Hamarneh, G., Möller, T.: Recovery of dynamic pet regions via simultaenous segmentation and deconvolution. In: Analysis of Functional Medical Image Data, pp. 33-40 (2008)

17. Wang, Q.: GMM-based hidden markov random field for color image and 3D volume segmentation. arXiv preprint arXiv:1212.4527 (2012)

18. Yazdanpanah, A., Hamarneh, G., Smith, B.R., Sarunic, M.V.: Segmentation of intra-retinal layers from optical coherence tomography images using an active contour approach. IEEE Trans. Med. Imag. 30(2), 484-496 (2011)

19. Zhang, Y., Brady, M., Smith, S.: Segmentation of brain MR images through a hidden markov random field model and the expectation-maximization algorithm. IEEE Trans. Med. Imag. 20(1), 45-57 (2001)

20. Zhu, H., Crabb, D.P., Schlottmann, P.G., Ho, T., Garway-Heath, D.F.: Floatingcanvas: quantification of 3D retinal structures from spectral-domain optical coherence tomography. Opt. Express 18(24), 24595-24610 (2010) 